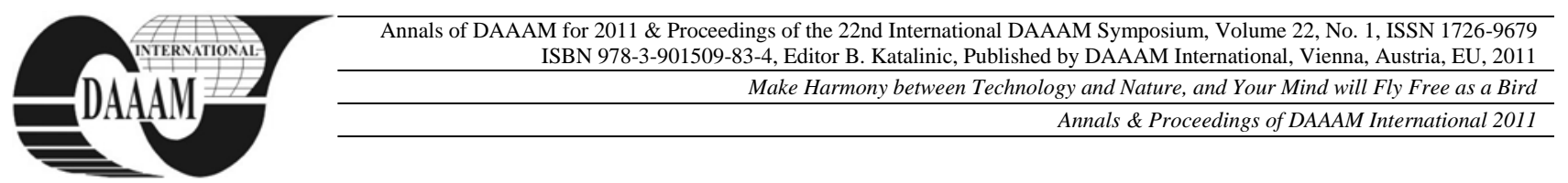

\title{
ROBOT POSE CORRECTION FOR OFFLINE PROGRAMMING OF INDUSTRIAL ROBOT SYSTEMS
}

\author{
BOZEK, P[avol]; TRNKA, K[amil]; KNAZIK, M[arek] \& ONDRIGA, M[artin]
}

\begin{abstract}
Installation of the device into plant prevents many hidden problems. The priority is to resolve them as soon as possible and in the best possible way. Today Robot and Production Facilities Offline Programming is considered to be the technological and temporal advantage not only in introducing new production technology, but also in application of the current production changes. On the one hand, this technology is termed by any real simulated state compared to the real world, on the other hand, the geometrical precision of the real environment in comparison with nominal values. Robot pose correction is an inseparable part of The Offline Programming, and this process can often reveal many hidden problems.
\end{abstract}

Key words: offline programming, robot, path, calibration, digital workplace, welding

\section{INTRODUCTION}

Model construction of manufacturing equipment, as well as the establishment of relevant robot programs by simulation system represents a true picture of reality. An absolute compliance with reality cannot be assumed. Ideally, it would be to load the robot program without any adaptation. However, there are essential differences between the computer model used to implement graphical simulation and real environment. Deviations may be caused by:

- $\quad$ errors in position of the workpiece and the environment due to the position of the robot, errors in tool precision with regard to the robot flange,

errors in the relative position of robot axes.

For these reasons, correction of the robot path is required, i.e. adapt the simulation to actual geometric conditions. It is an integrated process of modelling, measurements, numerical identification of robots real physical properties with the implementation of a new model (Stollmann, 2004).

\section{BASIC PRINCIPLES OF GEOMETRIC SIMULATION}

Geometric simulation is graphical three-dimensional computer model product and process geometry with full kinematics. Simulation is performed for each work station / cell and includes input-output flow of parts and components of technological equipment.

The simulation model is used throughout the life cycle of stations for proposition and verification of changes in geometric and process flow. Number of planned changes increases proportionally with the number of projects passing station / line and the number of ongoing projects which are in parallel with various activities on the same stations, but in different time frames (Hlavaty, 2004). These facts indicate that it is important to have proper models of all active stations. And that is the reason why it is necessary to use RCS-modules for the robotic station in order to achieve a decision based on correct analysis.

During installation, the robot is measured by the exact position (configuration of cell) in relation to the beginning of the coordinate system of the final product. These values are used in robot control module, but in the simulation model, there must be used nominal data according to the layout.

Considering the installation tolerances, it must be set a maximum permissible deviation of the robot movement and rotation. The same maximum deviation is applicable for the external TCP. As all target locations of the robot are determined in the workpiece coordinate system, deviations will only be reflected in values representing joints positions of the robot and not in its absolute position.

\section{OFFLINE PROGRAMMING IN WORKPIECE COORDINATE SYSTEM}

The aim of most manufacturers is that all programming of robots will run in offline. This allows to make quick changes in production (about tens of hours), to run coming models production and to reach full production with high quality within a few weeks (Pivarciová \& Sipos, 2006).

It is appropriate and simple to program robots in workpiece coordinates. Workpiece coordinate system is usually used in the CAD and CAPE Systems (e.g. Catia, Robcad). In order to use the coordinates of these systems and transfer them into robots, i.e. programmed them off-line, robots must be programmed in the coordinate system of the workpiece. It also facilitates the planning of paths for all the appropriate parts, if e.g. any used pose or path has only one coordinate set. Programming in the coordinate system of a body is a method that enables a quick and easy balancing pose and path among different robots and stations.

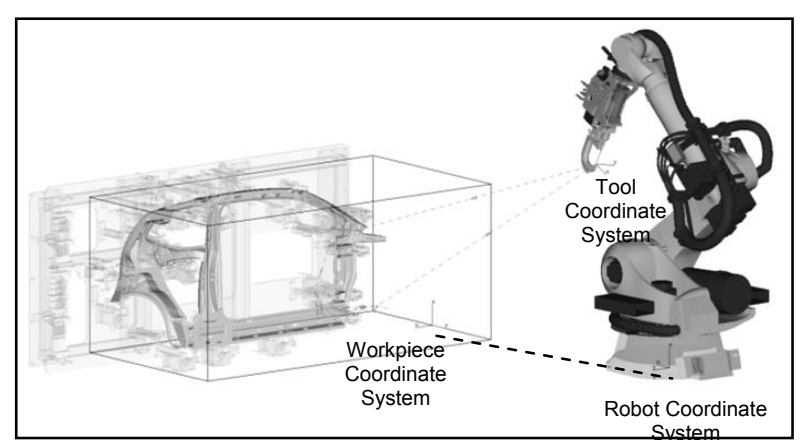

Fig. 1. Workpiece coordinate system and robot coordinate system

\section{TCP MEASUREMENT PROPOSAL AND WORKPIECE CALIBRATION}

Calibration has the most important influence on the acceptability of offline programming as only in case the virtual 
environment can be precisely mapped into the real environment, we can use automatically generated program in practice (Schlempp, 2005).

It is necessary to measure the exact position of the workpiece in relation to the robot before calibration. For calibration purposes is sufficient to measure and preset 3 coordinates, of which max. 2 may be located on the same line. It is important to point out that the 3 coordinates must have an equivalent in the virtual environment, which must be exactly identified.

The main problem by offline programs implementation is robot tolerances. Due to these factors is good for accuracy of measurement to choose also points, where configuration of joints does not reach extreme position. By creating items in a virtual environment, this effect appears to be a real mistake. (Karavaev et al, 2008). The tolerance for uploaded via locations is $\pm 1 \mathrm{~mm}$ and \pm 0.2 degrees. If there are deviations existing among the nominal locations and the uploaded program, there different cases which require different solutions may occur:

1) All uploaded locations are ok except via locations which are not in tolerance;

Solution: Update of via locations in the cell.

2) All the uploaded locations have a constant deviation;

Solution: Check the accuracy of robot in relation to mechanical installation requirements for functional packages and related objects. In case the solution is not found or it is time consuming and expensive, we can use calibration.

3) random deviations of uploaded locations;

Solution: In case that no other solution (similar to point 2) is found, it may be a geometric inaccuracy of the tool, or fixture.

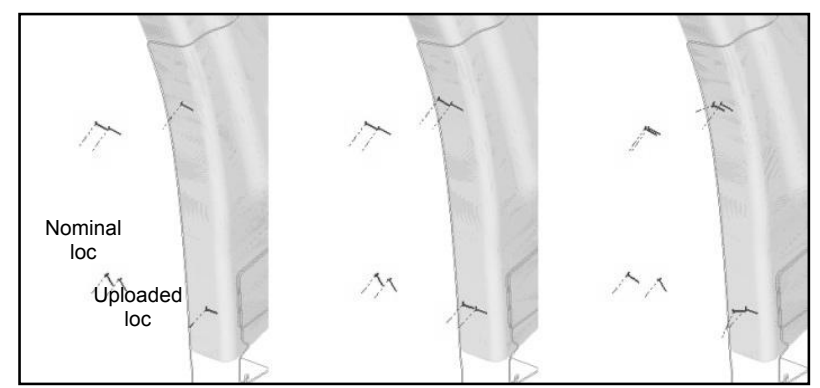

Fig. 2. From the left: case 1 OK to update / case 2 NOK to update | case 3 NOK to update

Generally we distinguish among the 3 calibration methods (Schlempp, 2005):

device calibration;

a) robot is calibrated relative to the position of the fixture where the number of robots is associated to one fixture;

b) fixture is calibrated in case the number of fixtures is associated to one robot;

- tool calibration by production conditional deviations of tool from nominal values;

- robot calibration by mathematical correction of positional error.

By offline programs implementation is the device or workpiece calibration used mostly. As mentioned above, since the offline programs are created in workpiece coordinate system, the calibration is triggered by a linear transformation of calibration pairs to put the origin of the workpiece coordinate system. In fact, his position does not change either in simulation or in real world. New values of its origin just offset mismatch between robot working offline locations toward reality. The obtained value replaces the nominal value set by simulation.

The advantage of the method described is, that there is no change in coordinates of working locations, but the whole coordinate system is moved in order to obtain as same values of robot joints rotation after the program is uploaded as by the simulation, in the same time it is needed to keep the nominal value of working point coordinates of a particular technology

\section{CONCLUSION}

To support the research target we have to improve the part of the methodology via an accurate kinematics model with easily identifiable characteristics. This is secured by offline programming primarily in the workpiece coordinate system. Calibration may still be a long-term process and its outcome is not always accurate (Zahorova \& Benes, 2008).

Mostly it is caused by measurement errors and wrong setup of tool center point. Further errors may occur by the introduction of the nominal load during production. This may be due to the excessive load of robot during the measurement. Therefore, it is preferable to carry out measurements by realload robot, which requires the use of different technologies (laser measurement, optical sensors, etc.). It is suitable to deal with a different implementation of calibration, which is often time consuming and thus suppresses the primary benefits of offline programming.

One option would be to implement the calibration functionality directly into the robot controller, while the actual standard of measurement, which are nominal values measured locations in the virtual environment, can be uploaded first. It would be possible to evaluate calibration results without downloading calibration path immediately after the measurements (Bubenik et.al, 2004).

In the future this would significantly speed up the calibration process, which is often limited by the absence of robot offline programmer directly by on-site installation.

\section{ACKNOWLEDGEMENTS}

The contribution was elaborated within the research project KEGA project No. 3-7285-09 Contents Integration and Design of University Textbook "Specialised Robotic Systems" in Print and Interactive Modules for University of Technology in Zvolen, Trenčín University and Slovak University of Technology in Bratislava.

\section{REFERENCES}

Bubenik, P.; Bubenikova, E.; Korbel, P. \& Nagy, P. (2004). Information technologies for business practice. In: EDIS ŽU, ISBN 80-8070-288-8

Hlavaty, I. (2004). Notation auxiliary material according to the European standard. In: Proceedings of "Welding II in nuclear energy." Ostravice: TU Ostrava, Czech Welding Institute Ltd. Ostrava, 25 to 27 May 2004, pp.135 - 151 st ISBN 80-248-0598-7

Karavaev, Y.L.; Lekomtsev, P. \& Abramov, A.I. (2008). Comparative analysis of the force parameters for drilling plasticand brittle materials, In: Bulletin Izhevsk STU № 1, 2008, Izhevsk, pp.10-15, ISSN 1813-7903

Pivarciova, E. \& Sipos, L. (2006). Programming techniques, Zvolen: Technica IUniversityin Zvolen, 2006. 173 pp. 1364 2.edition. ISBN 80-228-1478-4

Schlempp, H. (2005). UGS eM-OLP und eM-Calibration mit VKRC 1/2, Tecnomatixe M-Workplace V7.12, pp. 3-19

Stollmann,V. (2004). 3D movable cable system with purposes automatics. In: Robtep-2004, Automation/Robotics in theory and practice, Vyšné Ružbachy, ISBN 80-8073-1349, s. $492-497$

Zahorova, V. \&- Benes, L. (2008) Prediction of mechanical properties of material. In Bokúvka, O. (ed.). Materials Engineering, Vol. 15, 2008, No.2a. Žilina, 5. květen. 2008, p. 41-46. ISSN 1335-0803 